\title{
Latin squares with maximal partial transversals of many lengths
}

\author{
Anthony B. Evans * \\ anthony. evans@wright . edu
}

\author{
Adam Mammoliti ${ }^{\dagger}$ \\ adam.mammoliti@monash.edu
}

\author{
Ian M. Wanless ${ }^{\dagger}$ \\ ian.wanless@monash.edu
}

\begin{abstract}
A partial transversal $T$ of a Latin square $L$ is a set of entries of $L$ in which each row, column and symbol is represented at most once. A partial transversal is maximal if it is not contained in a larger partial transversal. Any maximal partial transversal of a Latin square of order $n$ has size at least $\left\lceil\frac{n}{2}\right\rceil$ and at most $n$. We say that a Latin square is omniversal if it possesses a maximal partial transversal of all plausible sizes and is near-omniversal if it possesses a maximal partial transversal of all plausible sizes except one.

Evans (2019) showed that omniversal Latin squares of order $n$ exist for any odd $n \neq 3$. By extending this result, we show that an omniversal Latin square of order $n$ exists if and only if $n \notin\{3,4\}$ and $n \not \equiv 2(\bmod 4)$. Furthermore, we show that near-omniversal Latin squares exist for all orders $n \equiv 2(\bmod 4)$.

Finally, we show that no non-trivial finite group has an omniversal Cayley table, and only 15 finite groups have a near-omniversal Cayley table. In fact, as $n$ grows, Cayley tables of groups of order $n$ miss a constant fraction of the plausible sizes of maximal partial transversals. In the course of proving this, we partially solve the following interesting problem in combinatorial group theory. Suppose that we have two finite subsets $R, C \subseteq G$ of a group $G$ such that $|\{r c: r \in R, c \in C\}|=m$. How large do $|R|$ and $|C|$ need to be (in terms of $m$ ) to be certain that $R \subseteq x H$ and $C \subseteq H y$ for some subgroup $H$ of order $m$ in $G$, and $x, y \in G$ ?
\end{abstract}

\section{Introduction}

A Latin square $L$ of order $n$ is an $n \times n$ matrix containing $n$ symbols such that each row and each column contains one copy of each symbol. Such a matrix is equivalent to a set of triples $(r, c, s)$ where $s$ is the symbol in cell $(r, c)$. This viewpoint will be exploited by using set notation and terminology when dealing with Latin squares and their submatrices. The three coordinates of a triple will be referred to as its row, its column and its symbol, respectively.

A partial transversal $T$ of a Latin square $L$ is a set of triples of $L$ that contains at most one representative of each row, column and symbol of $L$. The length of $T$ is the number of triples in it. A partial transversal is maximal if it is not contained in a partial transversal of greater length. Clearly, a maximal partial transversal $T$ of a Latin square of order $n$ has length at most $n$. For a Latin square of

\footnotetext{
*Department of Mathematics and Statistics, Wright State University, Dayton, OH 45435, United States.

†School of Mathematics, Monash University, Clayton 3800, Australia.
} 
order $n$ a transversal is a partial transversal of length $n$ and a near-transversal is a partial transversal of length $n-1$. For a survey of results and applications of transversals and partial transversals, see [17]. The following folklore result characterises all possible lengths that a maximal partial transversal may have.

Lemma 1.1. If $\ell$ is the length of a maximal partial transversal of a Latin square of order $n$, then

$$
\left\lceil\frac{n}{2}\right\rceil \leqslant \ell \leqslant n
$$

Best et al. [2, Thm 12] showed for $n \geqslant 5$ that all values of $\ell$ consistent with (11) are achieved, provided the host Latin square is allowed to depend on $n$ and $\ell$. In this paper we explore a stronger condition where a fixed Latin square achieves maximal partial transversals for all lengths satisfying (1). More generally, we will consider Latin squares that possess maximal partial transversals of many different lengths. To do so, we make the following definitions. A Latin square of order $n$ will be called omniversal if it has maximal partial transversals of each length satisfying (11) and will be called near-omniversal if it has maximal partial transversals of all but one of the lengths that satisfy (1).

By constructing a special class of Latin squares, Evans [7] proved the following.

Theorem 1.1. There exists an omniversal Latin square of order $n$ for all odd $n \neq 3$.

By considering Latin squares of even order, we will determine the necessary and sufficient conditions for the existence of an omniversal Latin square of order $n$.

Theorem 1.2. There exists an omniversal Latin square of order $n$ if and only if $n \notin\{3,4\}$ and $n \not \equiv 2$ $(\bmod 4)$.

One can easily see that every Latin square of order 3 is near-omniversal and that no omniversal or near-omniversal Latin squares of order 4 exist. All Latin squares of order 4 are equivalent to the Cayley table of a group and $\mathbb{Z}_{2} \times \mathbb{Z}_{2}$ has only transversals, while $\mathbb{Z}_{4}$ has only maximal near-transversals. For the remaining orders excluded in Theorem 1.2, we can at least show that there is a near-omniversal Latin square of order $n$.

Theorem 1.3. For any $n \equiv 2(\bmod 4)$, there exists a near-omniversal Latin square of order $n$.

We also consider Cayley tables of finite groups. We show that as the order grows, Cayley tables miss at least a constant fraction of the lengths permitted by Lemma 1.1. As a consequence, only the Cayley table of the trivial group is omniversal. We also obtain a complete catalogue of near-omniversal Cayley tables, where $\mathbb{Z}_{n}$ and $D_{n}$ denote the cyclic and dihedral groups of order $n$, respectively:

Theorem 1.4. No non-trivial group of finite order has an omniversal Cayley table. The only nearomniversal Cayley tables are those of $\mathbb{Z}_{2}, \mathbb{Z}_{3}, \mathbb{Z}_{5}, \mathbb{Z}_{6}, D_{6}, D_{8}$ and the non-abelian groups of order 16 .

Evidently, there is no hope of finding omniversal Cayley tables of finite groups. However, our strategy for constructing omniversal Latin squares will be to modify a Cayley table very slightly. Specifically, we will "turn an intercalate", meaning that we replace a $2 \times 2$ subsquare by the other possible subsquare on the same symbols. Note that by a subsquare, we mean a submatrix that is itself a Latin square. One other piece of terminology we will need is this: We say that two Latin squares are isotopic if one can be obtained from the other by applying three possibly distinct bijections to the rows, columns and symbols respectively.

The paper is organised as follows. We will prove Theorem 1.2 in Section 2 , by considering the cases when $n$ is 0 and 2 modulo 4 separately. The proof of Theorem 1.3 will be given in Section 3 , Section 4 includes preliminary results required for the proof of Theorem 1.4 but which may be of independent interest in the study of Cayley tables of groups. In particular, we look at how much of a subsquare needs to be present before we know the whole subsquare is present. The proof of Theorem 1.4 itself is presented in Section 5 . 


\section{Existence of omniversal Latin squares}

In this section we will prove Theorem 1.2, By Theorem 1.1, we only need to consider the case when $n$ is even. We will consider the cases when $n$ is 0 and 2 modulo 4 separately. We first show the non-existence of omniversal Latin squares of order 2 modulo 4. We then show the existence of omniversal Latin squares of any order $n \equiv 0(\bmod 4)$, except $n=4$.

Theorem 2.1. Let $L$ be a Latin square of order $4 m+2$ for some integer $m \geqslant 0$. Then either $L$ does not possess a transversal or it does not possess a maximal partial transversal of length $2 m+1$. In particular, $L$ is not omniversal.

Proof. Aiming for a contradiction, suppose that $L$ has both a maximal partial transversal $T$ of length $2 m+1$ and a transversal $T^{\prime}$. Let $A$ be the submatrix of $L$ consisting of the triples that do not share a row or column with any element of $T$. Without loss of generality, we may then assume that $L$ is partitioned into four square blocks $A, B, C, D$, each of order $2 m+1$ as shown:

$$
L=\left(\begin{array}{l|l}
A & B \\
\hline C & D
\end{array}\right) .
$$

As $T$ cannot be extended to a longer partial transversal, every symbol in $A$ is contained in $T$. It follows quickly that $A, B, C$, and $D$ are subsquares of $L$, with $A$ and $D$ on a symbol set $S_{1}$, and $B$ and $C$ on a symbol set $S_{2}$, where $S_{1} \cap S_{2}=\varnothing$.

The transversal $T^{\prime}$ of $L$ contains, say, $t$ triples from $A$. It follows that $T^{\prime}$ contains $2 m+1-t$ triples from each of $B$ and $C$, and $t$ triples from $D$. On the other hand, $T^{\prime}$ must contain each of the $2 m+1$ symbols in $S_{1}$ exactly once. That means that $2 t=2 m+1$, an impossibility from which the result follows.

As mentioned in the introduction, no Latin square of order 4 is omniversal (or even near-omniversal). There are Latin squares of order 8 that are omniversal. For example, any Latin square obtained from the Cayley table for the group $\mathbb{Z}_{2}^{3}$ by turning an intercalate is omniversal. Figure 1 depicts maximal partial transversals of such a Latin square of each possible length, shown by the blue shaded entries.

We will generalise the construction in Figure 1 to construct omniversal Latin squares of any order that is a multiple of 4 and at least 8 . Let $n=8 m+4 q$, where $q \in\{0,1\}$ and $m>0$. Write $G=\mathbb{Z}_{2}^{2} \times \mathbb{Z}_{2 m+q}$ multiplicatively as $\left\langle x, y, z: 1=x^{2 m+q}=y^{2}=z^{2}, x y=y x, x z=z x, y z=z y\right\rangle$. Let $H=\langle x\rangle$ be the subgroup of $G$ generated by $x$. Throughout this section, we will make frequent (implicit) use of the fact that $G$ is a disjoint union of cosets $G=H \cup y H \cup z H \cup y z H$.

Let $L_{8 m+4 q}$ be the Cayley table for $\mathbb{Z}_{2}^{2} \times \mathbb{Z}_{2 m+q}$. Let $L_{8 m+4 q}^{*}$ be the Latin square formed from $L_{8 m+4 q}$ by turning the intercalate with cells $(1,1),(1, y),(y, 1)$ and $(y, y)$, i.e.,

$$
L_{8 m+4 q}^{*}=\left(L_{8 m+4 q} \backslash\{(1,1,1),(1, y, y),(y, 1, y),(y, y, 1)\}\right) \cup\{(1,1, y),(1, y, 1),(y, 1,1),(y, y, y)\} .
$$

The Latin square repeated in Figure 1 is (isotopic to) $L_{8}^{*}$, with the turned intercalate marked by red crosshatching in the first copy. We will show that $L_{8 m+4 q}^{*}$ is omniversal, but first note the following theorem, which we will use in the proof. It follows from the recent proof of the Hall-Paige conjecture (see [3, 6, 17]).

Theorem 2.2. Let $G$ be a finite group and $L_{G}$ be the Cayley table for $G$. Then the following are equivalent.

(i) $L_{G}$ can be decomposed into disjoint transversals.

(ii) The Sylow 2-subgroups of $G$ are trivial or non-cyclic. 


\begin{tabular}{|llllllll|}
\hline 2 & 1 & 0 & 3 & 4 & 5 & 6 & 7 \\
1 & 0 & 3 & 2 & 5 & 4 & 7 & 6 \\
0 & 3 & 2 & 1 & 6 & 7 & 4 & 5 \\
3 & 2 & 1 & 0 & 7 & 6 & 5 & 4 \\
4 & 5 & 6 & 7 & 0 & 1 & 2 & 3 \\
5 & 4 & 7 & 6 & 1 & 0 & 3 & 2 \\
6 & 7 & 4 & 5 & 2 & 3 & 0 & 1 \\
7 & 6 & 5 & 4 & 3 & 2 & 1 & 0 \\
\hline
\end{tabular}

\begin{tabular}{|llll:llll|}
\hline 2 & 1 & 0 & 3 & 4 & 5 & 6 & 7 \\
1 & 0 & 3 & 2 & 5 & 4 & 7 & 6 \\
0 & 3 & 2 & 1 & 6 & 7 & 4 & 5 \\
3 & 2 & 1 & 0 & 7 & 6 & 5 & 4 \\
\hdashline 4 & 5 & 6 & 7 & 0 & 1 & 2 & 3 \\
5 & 4 & 7 & 6 & 1 & 0 & 3 & 2 \\
6 & 7 & 4 & 5 & 2 & 3 & 0 & 1 \\
7 & 6 & 5 & 4 & 3 & 2 & 1 & 0 \\
\hline
\end{tabular}

\begin{tabular}{|llll:llll|}
\hline 2 & 1 & 0 & 3 & 4 & 5 & 6 & 7 \\
1 & 0 & 3 & 2 & 5 & 4 & 7 & 6 \\
0 & 3 & 2 & 1 & 6 & 7 & 4 & 5 \\
3 & 2 & 1 & 0 & 7 & 6 & 5 & 4 \\
\hdashline 4 & 5 & 6 & 7 & 0 & 1 & 2 & 3 \\
5 & 4 & 7 & 6 & 1 & 0 & 3 & 2 \\
6 & 7 & 4 & 5 & 2 & 3 & 0 & 1 \\
7 & 6 & 5 & 4 & 3 & 2 & 1 & 0 \\
\hline
\end{tabular}

\begin{tabular}{|llll:llll|}
\hline 2 & 1 & 0 & 3 & 4 & 5 & 6 & 7 \\
1 & 0 & 3 & 2 & 5 & 4 & 7 & 6 \\
0 & 3 & 2 & 1 & 6 & 7 & 4 & 5 \\
3 & 2 & 1 & 0 & 7 & 6 & 5 & 4 \\
4 & 5 & 6 & 7 & 0 & 1 & 2 & 3 \\
5 & 4 & 7 & 6 & 1 & 0 & 3 & 2 \\
6 & 7 & 4 & 5 & 2 & 3 & 0 & 1 \\
7 & 6 & 5 & 4 & 3 & 2 & 1 & 0 \\
\hline
\end{tabular}

\begin{tabular}{|llll:llll|}
\hline 2 & 1 & 0 & 3 & 4 & 5 & 6 & 7 \\
1 & 0 & 3 & 2 & 5 & 4 & 7 & 6 \\
0 & 3 & 2 & 1 & 6 & 7 & 4 & 5 \\
3 & 2 & 1 & 0 & 7 & 6 & 5 & 4 \\
4 & 5 & 6 & 7 & 0 & 1 & 2 & 3 \\
5 & 4 & 7 & 6 & 1 & 0 & 3 & 2 \\
6 & 7 & 4 & 5 & 2 & 3 & 0 & 1 \\
7 & 6 & 5 & 4 & 3 & 2 & 1 & 0 \\
\hline
\end{tabular}

Figure 1: Maximal partial transversals of an omniversal Latin square of order 8.

Throughout the proof of our next theorem, we say that a set of elements $S$ avoids a row, column, or symbol $g$ if no element of $S$ is in row $g$, column $g$ or has symbol $g$, respectively. Similarly, a set of triples $S$ avoids a cell $(r, c)$ if $S$ contains no triple $(r, c, s)$ for any symbol $s$.

Theorem 2.3. The Latin square $L_{8 m+4 q}^{*}$ is omniversal for all $m>0$ and $q \in\{0,1\}$.

Proof. First we show that $L_{8 m+4 q}^{*}$ has a transversal and a maximal near-transversal. Clearly, the Sylow 2-subgroups of $G=\mathbb{Z}_{2}^{2} \times \mathbb{Z}_{2 m+q}$ are non-cyclic. Thus by Theorem $2.2, L_{8 m+4 q}$ can be decomposed into disjoint transversals. Therefore, since $L_{8 m+4 q}$ has order at least 8, at least one transversal $T$ of $L_{8 m+4 q}$ will avoid the cells $(1,1),(1, y),(y, 1)$ and $(y, y)$. Hence, $T$ is also a transversal of $L_{8 m+4 q}^{*}$. On the other hand, if $T^{\prime}$ is a transversal of $L_{8 m+4 q}$ which hits the cell $(1,1)$ but none of $(1, y),(y, 1)$ and $(y, y)$, then $T^{\prime} \backslash\{(1,1,1)\}$ must be a maximal near-transversal of $L_{8 m+4 q}^{*}$. As $L_{8 m+4 q}$ can be decomposed into disjoint transversals and any pair of the triples $(1,1,1),(1, y, y),(y, 1, y)$ and $(y, y, 1)$ share a row, column or symbol, such a transversal $T^{\prime}$ of $L_{8 m+4 q}$ exists and hence $L_{8 m+4 q}^{*}$ has a maximal near-transversal.

Now we show that $L_{8 m}^{*}$ has a maximal partial transversal of length $4 \mathrm{~m}$. Consider the subsquare $S$ of $L_{8 m}$ with rows and columns indexed by $z H \cup y z H$. As $S$ avoids the cells $(1,1),(1, y),(y, 1)$ and $(y, y)$, we see that $S$ is also a subsquare of $L_{8 m}^{*}$. Moreover, $z H \cup y z H$ is the coset $z\langle x, y\rangle$ of $G$, so $S$ is isotopic to the Cayley table for $\mathbb{Z}_{2} \times \mathbb{Z}_{2 m}$. Hence as $\mathbb{Z}_{2} \times \mathbb{Z}_{2 m}$ has a non-cyclic Sylow 2-group, $S$ has a transversal $T$, by Theorem 2.2 . It follows that $T$ is a partial transversal of $L_{8 m}^{*}$ of length $4 m$. As the rows and columns of $T$ are $z H \cup y z H$ and $T$ contains every symbol in $H \cup y H$, we can extend $T$ to a longer partial transversal only if there is $(r, c, s) \in L_{8 m}^{*}$ such that $r, c \in H \cup y H$ and $s \in z H \cup y z H$. However, every cell in rows and columns $H \cup y H$ of $L_{8 m}^{*}$ contains a symbol in $H \cup y H$. Thus, $T$ is a maximal partial transversal of length $4 \mathrm{~m}$.

We also show that $L_{8 m+4}^{*}$ has a maximal partial transversal of length $8 m+2$. Let

$$
T=\left\{\left(x^{i}, x^{i}, x^{2 i}\right),\left(x^{i} z, x^{i} y z, x^{2 i} y\right),\left(x^{i} y z, x^{i} y, x^{2 i} z\right),\left(x^{i} y, x^{i+1} z, x^{2 i+1} y z\right): 0 \leqslant i \leqslant 2 m\right\} .
$$


Clearly $T$ is a partial transversal of $L_{8 m+4}^{*}$ of length $8 m$ that avoids rows in $\{1, z, y z, y\}$, columns in $\{1, y z, y, x z\}$ and symbols in $\{1, y, z, x y z\}$. Therefore, $T \cup\{(1,1, y),(y, y z, z)\}$ is a maximal partial transversal of $L_{8 m+4}^{*}$ of length $8 m+2$, since there is no element of $L_{8 m+4}^{*}$ in row $r \in\{z, y z\}$ and column $c \in\{y, x z\}$ with a symbol in $\{1, x y z\}$.

Given what we have shown already, to prove the theorem it suffices to show that $L_{8 m+4 q}^{*}$ has a maximal partial transversal of each length $4 m+2 q+k$ for $1-q \leqslant k \leqslant 4 m+q-2$ (the upper bound for $k$ can be checked by considering the two possible values for $q$ ). Our constructions for partial transversals of these lengths will be in terms of $k$ and auxiliary parameters $w, v$ and $j$. Precise values for these parameters will be specified subsequently. At this stage we merely stipulate that $j$ is an integer satisfying $0 \leqslant j \leqslant 2 m-1$ and that $\{v, w\}=\{y, z\}$, where $x, y, z$ are the generators for $G$.

Let $U_{w}$ be the partial transversal of $L_{8 m+4 q}^{*}\left(\right.$ and $\left.L_{8 m+4 q}\right)$ defined by

$$
\begin{gathered}
U_{w}=\left\{\left(x^{i}, x^{i}, x^{2 i}\right): 1 \leqslant i \leqslant m-1+q\right\} \cup\left\{\left(w x^{i+1}, w x^{i}, x^{2 i+1}\right): 0 \leqslant i \leqslant m-1\right\} \cup \\
\left\{\left(x^{m+q+i}, w x^{m+q+i}, w x^{2 i+q}\right): 0 \leqslant i \leqslant m-1\right\} \cup\left\{\left(w x^{m+1+q+i}, x^{m+q+i}, w x^{2 i+q+1}\right): 0 \leqslant i \leqslant m-1\right\} .
\end{gathered}
$$

For $u \in G$ let $\rho_{u}$ and $\gamma_{u}$ be the functions defined by

$$
\rho_{u}(r, c, r c)=(u r, c, u r c) \quad \text { and } \quad \gamma_{u}(r, c, r c)=(r, c u, r c u)
$$

for all $(r, c, r c) \in L_{8 m+4 q}^{*}$. Also, for $u \in G \backslash H$ let $\sigma_{u}$ be the function defined by

$$
\sigma_{u}(r, c, r c)= \begin{cases}\left(x^{-m} y z r, c x^{m} y z, r c\right) & \text { if } r, c \in H \\ \left(x^{m} y z r, c x^{-m} y z, r c\right) & \text { if } r, c \in u H\end{cases}
$$

for any $(r, c, r c) \in L_{8 m+4 q}^{*}$ such that $r, c \in H \cup u H$ and $r c \in H$.

By permuting rows and columns, $L_{8 m+4 q}$ can be written in the form

$$
\left(\begin{array}{l|l}
A & B \\
\hline C & D
\end{array}\right)
$$

where the rows and columns of $A$ are indexed by $H \cup w H$, while the rows and columns of $D$ are indexed by $v H \cup v w H$. The rows and columns of $B$ and $C$ are indexed accordingly. One can easily check that $A$ and $D$ contain the symbols $H \cup w H$, while $B$ and $C$ contain the symbols $v H \cup v w H$.

Let $K$ be a $j$-subset of $U_{w}$ whose symbols are elements of $H \backslash\left\{x^{2 m}\right\}$. Clearly $U_{w}$ is contained in block $A$. It follows that $\gamma_{v}(K), \rho_{v w}(K)$ and $\sigma_{w}(K)$ are in blocks $B, C$ and $D$ respectively. We claim that

$$
U=\left(U_{w} \backslash K\right) \cup \gamma_{v}(K) \cup \rho_{v w}(K) \cup \sigma_{w}(K)
$$

is a partial transversal of $L_{8 m+4 q}^{*}$ (and $\left.L_{8 m+4 q}\right)$ of length $4 m-1+q+2 j$. Clearly $\left(U_{w} \backslash K\right), \gamma_{v}(K)$, $\rho_{v w}(K)$ and $\sigma_{w}(K)$ are each partial transversals.

If two elements of $U$ share a row, then they are from $\left(U_{w} \backslash K\right)$ and $\gamma_{v}(K)$, or from $\rho_{v w}(K)$ and $\sigma_{w}(K)$. The former is not possible by definition of $\gamma_{v}$ since the rows of elements of $\gamma_{v}(K)$ are exactly those of the elements of $K$. The latter occurs only if there are two elements in $K$ that are in rows $r$ and $r^{\prime}$, such that $r=r^{\prime} x^{m}$, yet no such elements of $K$ exist. If two elements of $U$ share a column, then they are from $\left(U_{w} \backslash K\right)$ and $\rho_{v w}(K)$, or from $\gamma_{v}(K)$ and $\sigma_{w}(K)$. The former is not possible as the columns of elements of $\gamma_{v}(K)$ are exactly those of the elements of $K$. The latter occurs only if there are two elements in $K$ that are in columns $c$ and $c^{\prime}$, such that $c=c^{\prime} w x^{m}$, yet no such elements of $K$ exist. If two elements of $U$ contain the same symbol, then they are from $\left(U_{w} \backslash K\right)$ and $\sigma_{w}(K)$, or from $\gamma_{v}(K)$ and $\rho_{v w}(K)$. The former is impossible as the symbols appearing in $\sigma_{v}(K)$ are exactly those appearing in $K$ and the latter is not possible as the elements of $\gamma_{v}(K)$ only contain symbols in $v H$, while the elements 
of $\rho_{v w}(K)$ contain only symbols in $v w H$. Therefore, $U$ is indeed a partial transversal of $L_{8 m+4 q}^{*}$. Finally, $U$ avoids the cells $(1,1),(1, y),(y, 1)$ and $(y, y)$ and has length $4 m-1+q+2 j$. We have thus proven our claim about $U$.

Suppose that $j=\left\lfloor\frac{k+q-1}{2}\right\rfloor$. Let $w=y$ if $k$ is even and $w=z$ if $k$ is odd. We extend $U$ as defined above to give

$$
T_{k, q}= \begin{cases}U \cup\left\{(1,1, y),\left(x^{m} y z, x^{m} y z, 1\right)\right\} & \text { if } k \text { is odd and } q=0, \\ U \cup\left\{(1,1, y),\left(x^{m+1} z, x^{m} z, 1\right),(y z, y, z)\right\} & \text { if } k \text { is odd and } q=1, \\ U \cup\left\{(1, z, z),\left(x^{m} y z, x^{m} y z, 1\right),(y z, 1, y z)\right\} & \text { if } k \text { is even and } q=0, \\ U \cup\left\{(1,1, y),\left(x^{m+1} y, x^{m} y, 1\right)\right\} & \text { if } k \text { is even and } q=1 .\end{cases}
$$

Now we show that $T_{k, q}$ is a maximal partial transversal of length $4 m+2 q+k$, as desired. It is easy to see that $T_{k, q}$ contains $4 m+2 q+k$ triples for each case of $k$ and $q$. We have already shown that $U$ is a partial transversal; we now argue that the extra triples added to $U$ do not share a row, column or symbol with any triple in $U$. It is easy to see that $U_{w}$ avoids row, column and symbol 1 when $q=0$ and rows 1 and $w x^{m+1}$, columns 1 and $w x^{m}$ and symbols 1 and $w$ when $q=1$. Let $g \in H \cup w H$. Note that $U$ contains an element that is in row $g$, column $g$ or has symbol $g$ if and only if an element of $U_{w}$ is in row $g$, column $g$ or has symbol $g$ respectively. It follows, for any $g \in H \cup w H$, that $T_{k, q}$ contains exactly one element in row $g$, one element in column $g$ and one element that has symbol $g$. By construction, $K$ avoids rows in $\left\{1, x^{m}, x^{-m}, w x^{m+1}\right\}$ columns in $\left\{1, x^{m}, w x^{m}\right\}$ and symbols in $\left\{1, x^{2 m}, w\right\}$. It follows that $\gamma_{v}(K) \cup \rho_{v w}(K) \cup \sigma_{w}(K)$ avoids rows $v w$ and $v w x^{-m}$, columns $v$ and $v w x^{m}$ and symbols $v$ and $v w$. Thus $T_{k, q}$ is a partial transversal for each case of $k$ and $q$.

Lastly, we show that $T_{k, q}$ is indeed maximal. For any $g \in H \cup w H$, we argued above that $T_{k, q}$ contains elements in row $g$, column $g$ and with symbol $g$. Thus, an element $(r, c, s) \in L_{8 m+4 q}^{*}$ could be added to $T_{k, q}$ to make a larger partial transversal only if $r, c, s \in v H \cup v w H$. For any $(r, c, s) \in L_{8 m+4 q}^{*}$ with $r, c \in v H \cup v w H$, we see that $s=r c \notin v H \cup v w H$, except possibly when $(r, c)$ is one of $(1,1),(1, y)$, $(y, 1)$ or $(y, y)$. Even in that case, $r, c, s \in v H \cup v w H$ only if $v=y$ and $(r, c, s)=(y, y, y)$. By definition, $v=y$ only if $k$ is odd, yet $(1,1, y) \in T_{k, q}$ when $k$ is odd. Hence $T_{k, q}$ is maximal, as claimed.

We can now prove Theorem 1.2 .

Proof of Theorem 1.2. By Theorems 1.1 and 2.3, there exists omniversal Latin squares of order $n$ when $n \neq 3$ is odd and $n \geqslant 8$ is divisible by 4, respectively. By Theorem 2.1, no omniversal Latin square of order 2 modulo 4 can exist. Finally, one can easily check that no omniversal Latin squares of orders 3 or 4 exist, concluding the proof.

\section{Near-omniversal Latin squares of order 2 modulo 4}

Given Theorem 2.1, it is natural to ask whether any Latin square of order 2 modulo 4 can be nearomniversal. The present section is devoted to proving this is indeed the case, namely, proving Theorem 1.3. Any Latin square of order 2 is clearly near-omniversal. In Figure 2 we present a near-omniversal Latin square of order 6 that is isotopic to the Cayley table of $\mathbb{Z}_{6}$ after turning an intercalate. Maximal partial transversals of this square of lengths 4,5 , and 6 are shown by the blue shading (again, the turned intercalate is indicated by red crosshatching).

Let $n=4 m+2$. The standard Cayley table of the group $\mathbb{Z}_{n}$ has the symbol $i+j(\bmod n)$ in cell $(i, j)$ of the table, for all $i, j \in\{0,1, \ldots, n-1\}$. An isotopic form of the Cayley table, which highlights the subgroup of index 2 , is the Latin square

$$
\left(\begin{array}{l|l}
A & B \\
\hline C & D
\end{array}\right),
$$




\begin{tabular}{|lll:lll|}
\hline 3 & 2 & 4 & 1 & 0 & 5 \\
2 & 4 & 0 & 3 & 5 & 1 \\
4 & 0 & 2 & 5 & 1 & 3 \\
\hdashline 1 & 3 & 5 & 2 & 4 & 0 \\
0 & 5 & 1 & 4 & 3 & 2 \\
5 & 1 & 3 & 0 & 2 & 4 \\
\hline
\end{tabular}

\begin{tabular}{|llllll|}
\hline 3 & 2 & 4 & 1 & 0 & 5 \\
2 & 4 & 0 & 3 & 5 & 1 \\
4 & 0 & 2 & 5 & 1 & 3 \\
1 & 3 & 5 & 2 & 4 & 0 \\
0 & 5 & 1 & 4 & 3 & 2 \\
5 & 1 & 3 & 0 & 2 & 4 \\
\hline
\end{tabular}

\begin{tabular}{|lll:lll|}
\hline 3 & 2 & 4 & 1 & 0 & 5 \\
2 & 4 & 0 & 3 & 5 & 1 \\
4 & 0 & 2 & 5 & 1 & 3 \\
\hdashline 1 & 3 & 5 & 2 & 4 & 0 \\
0 & 5 & 1 & 4 & 3 & 2 \\
5 & 1 & 3 & 0 & 2 & 4 \\
\hline
\end{tabular}

Figure 2: Maximal partial transversals in a near-omniversal Latin square of order 6.

where $A, B, C$, and $D$ are Latin squares of order $2 m+1$ with rows and columns indexed by $\mathbb{Z}_{2 m+1}$ and entries

$$
\begin{aligned}
& A=\left(a_{i j}\right)=2(i+j) \quad(\bmod 4 m+2), \\
& B=\left(b_{i j}\right)=2(i+j)+1 \quad(\bmod 4 m+2), \\
& C=\left(c_{i j}\right)=2(i+j)+1 \quad(\bmod 4 m+2),
\end{aligned}
$$

and

$$
D=\left(d_{i j}\right)=2(i+j)+2 \quad(\bmod 4 m+2),
$$

for all $i, j \in\{0,1, \ldots, 2 m\}$. Let $M_{4 m+2}^{*}$ denote the Latin square obtained from $M_{4 m+2}$ by changing four entries, i.e., turning an intercalate, by setting

$$
a_{00}=2 m+1=d_{m m} \quad \text { and } \quad b_{0 m}=0=c_{m 0} .
$$

The Latin square $M_{6}^{*}$ is depicted in Figure 2. We will prove that $M_{4 m+2}^{*}$ is near-omniversal, but first we require the following two lemmas.

Lemma 3.1. Let $a \in\{0, \ldots, 4 m+1\}$. Then the equation $4 i+a \equiv 0(\bmod 4 m+2)$ has a solution in $\{1, \ldots, m\}$ if and only if $a \equiv 2(\bmod 4)$, in which case there is a unique solution $i=m-\frac{a-2}{4}$.

Proof. If there is an $i \in\{1, \ldots, m\}$ such that $4 i \equiv-a(\bmod 4 m+2)$, then $4 i=4 m+2-a$, as $0<4 i<4 m+2$. Consequently, $a$ must be 2 modulo 4 and $i=m-\frac{a-2}{4}$. Conversely, if $a \equiv 2(\bmod 4)$, then $i=m-\frac{a-2}{4} \in\{1, \ldots, m\}$ and satisfies $4 i+a \equiv 0(\bmod 4 m+2)$.

Lemma 3.2. The equation $4 i+2 \equiv 4 j(\bmod 4 m+2)$ has no solutions $(i, j)$ that satisfy $|i-j| \leqslant m-1$.

Proof. The solutions to $4 i+2 \equiv 4 j(\bmod 4 m+2)$ are any $i$ and $j$ such that $4(i-j)+2 \equiv 0(\bmod 4 m+2)$. If $|i-j| \leqslant m-1$, then

$$
-4 m+6 \leqslant 4(i-j)+2 \leqslant 4 m-2 .
$$

Therefore, $4(i-j)+2 \equiv 0(\bmod 4 m+2)$ only if $4(i-j)+2=0$, yet this is impossible, as the left hand side is 2 modulo 4 while the right hand side is 0 modulo 4 .

We will now prove that $M_{4 m+2}^{*}$ is near-omniversal, thus proving Theorem 1.3 .

Theorem 3.1. The Latin square $M_{4 m+2}^{*}$ is near-omniversal for all $m \geqslant 0$. For $m \geqslant 1$, it does not possess a maximal partial transversal of length $2 m+1$. 
Proof. The theorem is clearly true for $m=0$. Hence, we will assume that $m \geqslant 1$ and construct a maximal partial transversal of each valid length except $2 m+1$ in $M_{4 m+2}^{*}$. Note that in the case when $m=1$, these maximal partial transversals are exactly those depicted in Figure 2. Let $H=\langle 2\rangle$ be the subgroup of $\mathbb{Z}_{4 m+2}$ of index 2 ; thus $\mathbb{Z}_{4 m+2}=H \cup(H+1)$.

First we construct a maximal partial transversal of length $2 m+2 k+3$, for each $k \in\{0,1, \ldots, m-1\}$. Let $T_{A}$ consist of the triples $(i, i+k+1,4 i+2 k+2)$, for $0 \leqslant i \leqslant k$, from $A$ and $T_{D}$ consist of the triples $(j+k+1, j, 4 j+2 k+4)$, for $0 \leqslant j \leqslant k$, from $D$. It follows from Lemma 3.2 that $4 i \equiv 4 j+2$ $(\bmod 4 m+2)$ has no solutions for $i, j \in\{0, \ldots, k\}$ and hence the symbols in $T_{A}$ and $T_{D}$ are distinct. Let $T_{C}$ consist of the triples $(i, i, 4 i+1)$ for $0 \leqslant i \leqslant k$ from $C$ and $T_{B}$ consist of the triples $(j, j, 4 j+1)$ for $k+1 \leqslant j \leqslant 2 m$ from $B$; the symbols in the triples in $T_{B} \cup T_{C}$ are precisely the elements of $H+1$. Thus, as the only triples that are not in a row or column of a triple in $T=T_{A} \cup T_{B} \cup T_{C} \cup T_{D}$ are from $C$ and no triple from $C$ has a symbol from $H \backslash\{0\}$ (and even the symbol 0 is unavailable given that it only occurs in the first column of $C$ and $T_{C}$ includes a triple in that column), it follows that $T$ cannot be extended to a longer partial transversal. Therefore, $T$ is a maximal partial transversal of length $2 m+2 k+3$.

Next, we construct a maximal partial transversal of length $2 m+2 k+2$ for each $k \in\{0,1, \ldots, m-1\}$. Let $T_{C}$ consist of the triples $(i, i, 4 i+1)$ for $1 \leqslant i \leqslant k$ and $(m, 0,0)$ from $C$ and $T_{B}$ consist of the triples $(j, j, 4 j+1)$ for $k+1 \leqslant j \leqslant 2 m$ and $(0,0,1)$ from $B$. The symbols in the triples of $T_{B} \cup T_{C}$ consist of precisely the elements of $\{0\} \cup(H+1)$ and $T_{B} \cup T_{C}$ has length $2 m+2$. As no triple of $B \cup C$ contains a symbol in $H \backslash\{0\}$, we see that $T_{B} \cup T_{C}$ can only be extended to a longer partial transversal by adding triples of the submatrix $A^{\prime}$ of $A$ consisting of the triples $(i, j, 2 i+2 j)$ such that $i \in\{1, \ldots, k\}$ and $j \in\{k+1, \ldots, 2 m\}$; and the submatrix $D^{\prime}$ of $D$ consisting of the triples $(i, j, 2 i+2 j+2)$ such that $i \in(\{k+1, \ldots, 2 m\} \cup\{0\}) \backslash\{m\}$ and $j \in\{1, \ldots, k\}$. There are only $k$ rows in $A^{\prime}$ and $k$ columns in $D^{\prime}$, so at most $2 k$ triples of $A^{\prime} \cup D^{\prime}$ can form a partial transversal. Hence, if $T^{\prime}$ is a partial transversal of length $2 k$ of $A^{\prime} \cup D^{\prime}$ with symbols in $H \backslash\{0\}$, then $T_{B} \cup T_{C} \cup T^{\prime}$ is a maximal partial transversal of length $2 m+2 k+2$. We proceed by constructing such a partial transversal $T^{\prime}$, considering the cases when $m$ is even and odd separately.

First suppose that $m$ is even. Let $T_{A^{\prime}}$ consist of the triples $(i, i+m, 4 i+2 m)$, for $1 \leqslant i \leqslant k$, from $A^{\prime}$. Let $T_{D^{\prime}}$ consist of the triples $(j+m, j, 4 j+2 m+2)$ for $1 \leqslant j \leqslant \min \left\{k, \frac{m}{2}-1\right\}$ from $D^{\prime}$ and let $T_{D^{\prime}}^{\prime}$ consist of the triples $(j+m+2, j, 4 j+2 m+6)$ for $\frac{m}{2} \leqslant j \leqslant k$ from $D^{\prime}$. Note that $T_{D^{\prime}}^{\prime}$ is empty if $k \leqslant \frac{m}{2}-1$ and that the triple $(0, m-1,2 m)$ is in $T_{D^{\prime}}^{\prime}$ when $k=m-1$. We show that no symbol in $T^{\prime}=T_{A^{\prime}} \cup T_{D^{\prime}} \cup T_{D^{\prime}}^{\prime}$ is 0. By Lemma 3.1, the symbols in $T_{A^{\prime}}$ cannot be zero, given that $m$ is even. Similarly, Lemma 3.1 shows that $4 j+2 m+2=0$ for $j \in\{1, \ldots, k+1\}$ only when $j=\frac{m}{2}$. It follows that no symbol in $T_{D^{\prime}} \cup T_{D^{\prime}}^{\prime}$ is 0 . Finally we show that $T^{\prime}$ is a partial transversal. A symbol in $T_{A^{\prime}}$ is the same as one in $T_{D^{\prime}} \cup T_{D^{\prime}}^{\prime}$ only if $4 i \equiv 4 j+2$ for some $i \in\{1, \ldots, k\}$ and $j \in\{1, \ldots, k+1\}$, but, by Lemma 3.2, there is no such $i$ and $j$. Hence, the symbols in the triples of $T_{A^{\prime}}$ and $T_{D^{\prime}} \cup T_{D^{\prime}}^{\prime}$ are distinct, and so $T^{\prime}$ is a partial transversal of length $2 k$ of $A^{\prime} \cup D^{\prime}$ with symbols only in $H \backslash\{0\}$. Thus, $M_{4 m+2}^{*}$ has a maximal partial transversal of length $2 m+2 k+2$ when $m$ is even.

Now suppose that $m$ is odd. Let $T_{A^{\prime}}$ consist of the triples $(i, i+m, 4 i+2 m)$ for $1 \leqslant i \leqslant \min \left\{k, \frac{m-1}{2}\right\}$ and $T_{A^{\prime}}^{\prime}$ consist of the triples $(j, j+m+1,4 j+2 m+2)$ for $\frac{m+1}{2} \leqslant j \leqslant k$. Also let $T_{D^{\prime}}$ consist of the triples $(j+m, j, 4 j+2 m+2)$ for $1 \leqslant j \leqslant \min \left\{k, \frac{m-1}{2}\right\}$ and let $T_{D^{\prime}}^{\prime}$ consist of the triples $(i+m+1, i, 4 i+2 m+2)$ for $\frac{m+1}{2} \leqslant i \leqslant k$. Note that $T_{A^{\prime}}^{\prime}$ and $T_{D^{\prime}}^{\prime}$ are empty if $k \leqslant \frac{m-1}{2}$. First we show that no symbol in $T^{\prime}=T_{A^{\prime}} \cup T_{A}^{\prime} \cup T_{D^{\prime}} \cup T_{D^{\prime}}^{\prime}$ is 0 . Lemma 3.1 shows that $4 i+2 m \not \equiv 0$ for any $i \in\{1, \ldots, k+1\} \backslash\left\{\frac{m+1}{2}\right\}$, so no symbol in $T_{A^{\prime}} \cup T_{D^{\prime}}^{\prime}$ is 0 . Lemma 3.1 also shows that the symbols in $T_{A^{\prime}}^{\prime} \cup T_{D^{\prime}}$ cannot be 0 , since $m$ is odd. Finally we show that $T^{\prime}$ is a partial transversal. A symbol in $T_{A^{\prime}}^{\prime} \cup T_{D^{\prime}}$ is the same as one in $T_{A^{\prime}} \cup T_{D^{\prime}}^{\prime}$ only if $4 i=4 j+2$ for some $i \in\{1, \ldots, k+1\}$ and $j \in\{1, \ldots, k\}$, yet by Lemma 3.2 no such $i$ and $j$ exist. Hence, $T^{\prime}$ is a partial transversal of length $2 k$ with symbols only in $H \backslash\{0\}$. Therefore $M_{4 m+2}^{*}$ has a maximal partial transversal of length $2 m+2 k+2$ when $m$ is odd.

Thus far, we have established the existence of maximal partial transversals of $M_{4 m+2}^{*}$ of lengths 
$2 m+2, \ldots, 4 m+1$. The existence of a maximal partial transversal of length $4 m+2$, i.e., a transversal, follows from Theorem 2.3 in [7]. Finally, by Theorem [2.1, $M_{4 m+2}^{*}$ cannot have a maximal partial transversal of length $2 m+1$ as it has a transversal. This completes the proof.

\section{Extending partial subsquares}

A result from [7], which was useful when constructing omniversal Latin squares of odd order, showed that if a Cayley table of a finite cyclic group contains all but one row of a Latin square (of order at least 3) then it contains the whole of that square as a subsquare. We will need a more general version of this result in our proof of Theorem 1.4. However, we think the issue is interesting enough to treat in a separate section. Informally, how much of a Latin square do we need to see inside a Cayley table before we can be sure the whole square is there? One possible approach to making that question concrete is to consider the Hamming distance between Cayley tables (see e.g. [5, 10, 16]). However, we need a different approach, because the application we have in mind is when we encounter a submatrix which is surprisingly pure, in that it contains few symbols for its size. Under what conditions can we be sure such a submatrix is inside a subsquare on the same set of symbols? In particular, we will consider the following question. If $R$ is a submatrix of a Cayley table and $R$ contains $m$ symbols, then how large does $R$ need to be (in terms of $m$ ) to guarantee that $R$ can be extended to a subsquare of $L$ which contains only the $m$ symbols in $R$ ? We conjecture the following answer.

Conjecture 4.1. Let $L$ be a Cayley table of a group $G$. Suppose that $R$ is an $\alpha m \times \beta m$ submatrix of $L$ containing $m$ symbols, where $\alpha>\frac{1}{2}$ and $\beta>\frac{2}{3}$. Then $R$ is contained in an $m \times m$ subsquare of $L$.

We stress that whenever we refer, say, to a submatrix containing $m$ symbols, we mean that there are exactly $m$ different symbols (and not more) that appear within the submatrix. Those $m$ symbols may be repeated any number of times within the submatrix. We also note that in this section we are not assuming that our groups are finite, but we are looking at finite submatrices of their Cayley tables. We will prove Conjecture 4.1 is true for abelian groups, and prove a weakened version for non-abelian groups. We will also show that the conjecture is in some sense best possible.

Let $G$ be a group. For $X, Y \subseteq G$, and an element $g \in G$ we let $g X=\{g x: x \in X\}, Y g=\{y g: y \in$ $Y\}$ and $X Y=\{x y: x \in X, y \in Y\}$. When the group $G$ is abelian, we will use additive notation. That is, we will write $g X$ as $g+X$ and $X Y$ as $X+Y$ etc. The following examples show that the inequalities in Conjecture 4.1 cannot be strengthened.

Example 4.1. Let $G$ be a group and $H$ be a finite normal subgroup of $G$ such that some $g \in G \backslash H$ satisfies $g^{2} \notin H$. Let $L$ be the Cayley table for $G$ and $R$ be the $\frac{1}{2} m \times m$ submatrix of $L$ with rows indexed by $H$ and columns indexed by $H \cup g H$, where $m=2|H|$. Then $R$ cannot be extended to an $m \times m$ subsquare $R^{\prime}$ of $L$. To see this, note that $R$ includes the row and column indexed by the identity, and already includes the symbols in $H \cup g H$. Therefore $R^{\prime}$ must include the rows indexed by elements of $\mathrm{gH}$, which leads to it including the symbols in $g^{2} H$ as per Figure 3 (a). However, by assumption $g^{2} H \nsubseteq H \cup g H$.

Example 4.2. Let $G$ be a group and $H$ be a finite normal subgroup of $G$ such that some element $g \in G \backslash H$ satisfies $g^{2}, g^{3} \notin H$. Let $L$ be the Cayley table for $G$ and $R$ be the $\frac{2}{3} m \times \frac{2}{3} m$ submatrix with rows indexed by $H \cup g H$ and columns indexed by $H \cup g^{-1} H$, where $m=3|H|$. Then $R$ cannot be extended to an $m \times m$ subsquare of $L$. The proof is similar to the previous example and is illustrated in Figure $3(b)$. If $R$ does extend to a subsquare, then that subsquare must include columns indexed by elements of $g H$, and hence include the symbols in $g^{2} H$. However, by assumption, $g^{2} H \nsubseteq H \cup g H \cup g^{-1} H$. 


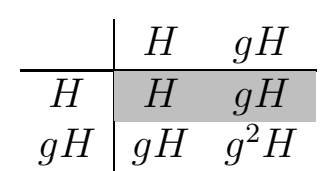

(a)

\begin{tabular}{c|ccc} 
& $H$ & $g^{-1} H$ & $g H$ \\
\hline$H$ & $H$ & $g^{-1} H$ & $g H$ \\
$g H$ & $g H$ & $H$ & $g^{2} H$
\end{tabular}

(b)

Figure 3: Examples showing Conjecture 4.1 is best possible. The shaded regions represent $R$.

The previous two examples showed submatrices of Cayley tables that could not be extended to subsquares of the Cayley table on the same set of symbols. One could also ask when can a submatrix $R$ of a Cayley table $L$ be extended to a Latin square, with only symbols in $R$, that is not necessarily a subsquare of $L$ ? Perhaps surprisingly, this is always possible.

Theorem 4.1. Let $R$ be any finite submatrix of a Cayley table. Suppose that $R$ contains $n$ symbols. Then $R$ can be embedded in some Latin square of order $n$.

Proof. A famous theorem of Ryser [15] gives necessary and sufficient conditions for a matrix to be embeddable in a Latin square of order $n$. Theorem 1 from [11] (see also [9]) shows that in a Cayley table these conditions are satisfied by any submatrix that contains $n$ symbols.

Next we prove Conjecture 4.1 for abelian groups. We will require the following result from additive combinatorics.

Theorem 4.2 (Kneser's Theorem [12]). Let $Z=X+Y$ for finite subsets $X, Y$ of an abelian group $G$. Then

$$
|Z| \geqslant|X|+|Y|-|H|
$$

for the subgroup $H=\{g \in G: g+Z=Z\}$.

Theorem 4.3. Let $L$ be a Cayley table of an abelian group $G$. Suppose that $R$ is an $\alpha m \times \beta m$ submatrix of $L$ containing $m$ symbols where $\alpha>\frac{1}{2}$ and $\beta>\frac{2}{3}$. Then $R$ is contained in an $m \times m$ subsquare of $L$.

Proof. Let $X$ and $Y$ be the indices of the rows and columns of $R$, respectively. Then the set $Z$ of symbols in $R$ is given by $Z=X+Y$. Let $H$ be the subgroup $\{g \in G: g+Z=Z\}$. Then $X^{\prime}=X+H$ and $Y^{\prime}=Y+H$ satisfy $X^{\prime}+Y^{\prime}=Z$. It follows that $S$, the submatrix of $L$ on rows $X^{\prime}$ and columns $Y^{\prime}$, contains only symbols in $Z$. As $X \subseteq X^{\prime}$ and $Y \subseteq Y^{\prime}$, we see that $R$ is contained in $S$ and so it suffices to show that $S$ is in fact an $m \times m$ subsquare of $L$.

As $H$ is a subgroup, $X^{\prime}=X^{\prime}+H$ and $Y^{\prime}=Y^{\prime}+H$. In particular, $x+H \subseteq X^{\prime}$ and $y+H \subseteq Y^{\prime}$ for all $x \in X$ and $y \in Y$. It follows that $X^{\prime}$ and $Y^{\prime}$ are each a union of cosets of $H$. Consequently, $Z$ is also the union of cosets of $H$. Let $\left|X^{\prime}\right|=\alpha^{\prime}|H|$ and $\left|Y^{\prime}\right|=\beta^{\prime}|H|$, i.e., $X^{\prime}$ is the union of $\alpha^{\prime}$ cosets of $H$ and $Y^{\prime}$ is the union of $\beta^{\prime}$ cosets of $H$. By Theorem 4.2,

$$
|Z| \geqslant\left|X^{\prime}\right|+\left|Y^{\prime}\right|-|H|=\left(\alpha^{\prime}+\beta^{\prime}-1\right)|H|
$$

First suppose that $|Z|>\left(\alpha^{\prime}+\beta^{\prime}-1\right)|H|$. As $Z$ is the union of cosets of $H$, if $|Z| \neq\left(\alpha^{\prime}+\beta^{\prime}-1\right)|H|$, then $|Z| \geqslant\left(\alpha^{\prime}+\beta^{\prime}\right)|H|$. Therefore,

$$
\alpha=\frac{|X|}{m} \leqslant \frac{\left|X^{\prime}\right|}{m} \leqslant \frac{\alpha^{\prime}|H|}{\left(\alpha^{\prime}+\beta^{\prime}\right)|H|}=\frac{\alpha^{\prime}}{\left(\alpha^{\prime}+\beta^{\prime}\right)}
$$

By a similar argument, $\beta \leqslant \frac{\beta^{\prime}}{\left(\alpha^{\prime}+\beta^{\prime}\right)}$. However, then $\alpha+\beta \leqslant 1$, contradicting the assumptions of the theorem. 
Thus, $|Z|=\left(\alpha^{\prime}+\beta^{\prime}-1\right)|H|$. By a similar argument to the above,

$$
\frac{1}{2}<\alpha \leqslant \frac{\alpha^{\prime}}{\alpha^{\prime}+\beta^{\prime}-1} \text { and } \quad \frac{2}{3}<\beta \leqslant \frac{\beta^{\prime}}{\alpha^{\prime}+\beta^{\prime}-1}
$$

Simplification yields the identities $\beta^{\prime}-1<\alpha^{\prime}$ and $2 \alpha^{\prime}-2<\beta^{\prime}$. As both $\alpha^{\prime}$ and $\beta^{\prime}$ are positive integers, we must have $\alpha^{\prime} \geqslant \beta^{\prime} \geqslant 2 \alpha^{\prime}-1$, from which it follows that $\beta^{\prime}=\alpha^{\prime}=1$. Hence, $\left|X^{\prime}\right|=\left|Y^{\prime}\right|=|Z|$ and $S$ is a $m \times m$ subsquare of $L$.

We now consider general groups. Unlike the abelian case, an analogue to Theorem 4.2 does not hold; counterexamples are given by Olson [13]. Instead, we will make use of the following result from [13].

Theorem 4.4. Let $Z=X Y$ for finite subsets $X, Y$ of a group $G$ such that $1 \in X$. Then either

(i) $X Z=Z$ or;

(ii) $|Z| \geqslant \frac{1}{2}|X|+|Y|$.

Using this result we can prove a weakened form of Conjecture 4.1 holds for all groups.

Theorem 4.5. Let $L$ be a Cayley table of a group $G$. Suppose that $R$ is an $\alpha m \times \beta m$ submatrix of $L$ with $m$ symbols, where $\frac{1}{2}<\alpha \leqslant \beta \leqslant 1$. If $\frac{\alpha}{2}+\beta>1$, then $R$ is contained in an $m \times m$ subsquare of $L$.

Proof. Let $X$ and $Y$ be the indices of the rows and columns of $R$, respectively, and $Z=X Y$ be the symbols in $R$. For any $g \in G, R$ is contained in an $m \times m$ subsquare of $L$ if and only if the submatrix of $L$ on rows $g X$ and columns $Y$ is contained in an $m \times m$ subsquare of $L$. Therefore without loss of generality, we can assume that $1 \in X$. Then by Theorem $4.4, Z=X Z$, since

$$
\frac{1}{2}|X|+|Y|=\left(\frac{\alpha}{2}+\beta\right) m>m=|Z| .
$$

Consequently, $Z=X^{i} Z$ for all $i \in \mathbb{Z}$ and so $Z=H Z$ for $H=\langle X\rangle$. Therefore as $1 \in X$, we have $Y \subseteq Z$ and so $R$ is contained in the submatrix $S$ of $L$ with rows indexed by $H$ and columns indexed by $Z$, which only contains symbols in $Z$. So we can complete the proof by showing that $S$ is in fact an $m \times m$ subsquare of $L$, for which it suffices to prove that $|Z|=|H|$. As $Z=H Z$, we see that $Z$ is the union of (right) cosets of $H$. On the other hand, $H \supseteq X$ and $|X|>\frac{1}{2}|Z|$. Hence, $Z$ must be in fact be a coset of $H$. It follows that $|H|=|Z|$, as required.

\section{Finite group tables}

In this section we consider the lengths of maximal partial transversals in the Cayley tables of finite groups. We find that Cayley tables get further and further from being omniversal as they get larger. This enables us to diagnose which Cayley tables are omniversal or near-omniversal. In particular, we prove Theorem 1.4.

First we state some preliminary results, starting with a well-known result about subsquares of Cayley tables (for a proof, see [4]).

Lemma 5.1. Let $S$ be a subsquare of the Cayley table of a group $G$ of order $n$. Then $G$ has a subgroup $H$ such that $S$ is formed by the rows indexed by $x H$ and columns indexed by Hy for some cosets $x H$ and $H y$ of $H$. In particular, $S$ must have order dividing $n$. 
In the case of abelian groups, we also have the following observation due to Belyavskaya and Russu [1] (see also [2, Lem.1]). A proof of this observation is implicit in earlier work of Paige [14]. Paige proved that a finite abelian group possesses a complete mapping if and only if its Sylow 2-subgroup is trivial or noncyclic (see also [6. Thm 3.9]), and a proper near complete mapping if and only if its Sylow 2-subgroup is nontrivial and cyclic (see also [6, Cor. 3.10 or Thm 15.2]). It is well-known that the Cayley table of a finite group has a transversal if and only if the group possesses a complete mapping, and a maximal near-transversal if and only if the group possesses a proper near complete mapping.

Theorem 5.1. No Cayley table of a finite abelian group has both a transversal and a maximal neartransversal.

By Theorem 2.2 we know exactly which Cayley tables of finite groups have transversals. This also tells us which Cayley tables of groups of order $n$ have maximal partial transversals of length $n / 2$. Such a partial transversal can only be achieved by taking a transversal of a subsquare of order $n / 2$. Hence Theorem 2.2 and Lemma 5.1 give us:

Corollary 5.1. The Cayley table of a group of order $n$ has a maximal partial transversal of length $n / 2$ if and only if it has an index 2 subgroup $H$ for which the Sylow 2-subgroups are trivial or non-cyclic.

In particular, the Cayley tables of all groups of order $n \equiv 2(\bmod 4)$ and no groups of order $n \equiv 4$ (mod 8) will have a maximal partial transversal of length $n / 2$.

When a Cayley table of a group does not have a transversal, it will have a maximal near-transversal, as a result of the following recent result of Goddyn and Halasz [8].

Theorem 5.2. The Cayley table of any finite group has a near-transversal.

Our first main result for this section shows that large groups are very far from being omniversal. Indeed, they miss at least a constant fraction of the lengths permitted by Lemma 1.1.

Theorem 5.3. Let $L$ be a Cayley table of a group $G$ of order $n$ and suppose that $L$ contains a maximal partial transversal $T$ of length $\ell<\frac{3}{5} n$. Then $n$ is even, $G$ contains a subgroup of index 2 and $\ell-\frac{n}{2}$ is even.

Proof. Let $R$ be the submatrix of $L$ that consists of the triples that do not share a row or column with any triple in $T$. Suppose that $R$ contains $m$ symbols. As $T$ is maximal, the symbols in $R$ must also be symbols in $T$ and, in particular, $m \leqslant \ell$. As $n-\ell>\frac{2}{3} \ell$ and $R$ is an $(n-\ell) \times(n-\ell)$ submatrix of $L$, Theorem 4.5 implies that $R$ is inside a subsquare $S$ of $L$ of order $m$ such that $n-\ell \leqslant m \leqslant \ell$. Now $n>m \geqslant n-\ell>\frac{2}{5} n$. So the only way to have $m \mid n$ as required by Lemma 5.1, is if $n=2 m$ and $G$ has an index 2 subgroup. It follows that $L$ takes the form

$$
\left(\begin{array}{l|l}
A & B \\
\hline C & S
\end{array}\right)
$$

where $A, S$ and $R$ have the same $m$-set of symbols. Let $w, x, y, z$ be the number of triples that $T$ includes from $A, B, C, S$ respectively. Since $T$ contains every symbol in $R$ it must contain exactly $m=w+z$ triples from $A \cup S$ and $\ell-m=x+y$ triples from $B \cup C$.

We claim that $x=y$, from which it will follow that $\ell-m$ is even, as required. Suppose on the contrary, that $x \neq y$. Assume that $x<y$ and $w \geqslant z$ (the cases when $x>y$ or $z>w$ work similarly). Then, since $x+w<y+w \leqslant m$, there must be a row $r$ of $A \cup B$ that is not represented in $T$. Of the $m$ triples in row $r$ of $B$, only $x+y=\ell-m$ of them have a symbol which occurs in $T$, and $x+z<\frac{1}{2} \ell$ of them have a column that is used in $T$. As $2 x+y+z<\frac{3}{2} \ell-m<m$, there is a triple in row $r$ that can be used to extend $T$. This contradiction of the maximality of $T$ completes the proof. 
In particular, Theorem 5.3 shows that groups with no index 2 subgroup (including all groups of odd order) miss all lengths $\ell$ in the range $\frac{1}{2} n \leqslant \ell<\frac{3}{5} n$. The inequality cannot be replaced with equality, as the following maximal partial transversal in the Cayley table of $\mathbb{Z}_{5}$ shows:

\begin{tabular}{|lllll|}
\hline 0 & 1 & 2 & 3 & 4 \\
1 & 2 & 3 & 4 & 0 \\
2 & 3 & 4 & 0 & 1 \\
3 & 4 & 0 & 1 & 2 \\
4 & 0 & 1 & 2 & 3 \\
\hline
\end{tabular}

Indeed, this example can be used to construct infinitely many examples for which the theorem is tight. Suppose that $G$ is a group of order $n$ which has a normal subgroup $N$ for which $G / N \cong \mathbb{Z}_{5}$ and the Cayley table of $N$ has a transversal. Then by combining transversals of the 3 blocks of the Cayley table of $G$ that are mapped to the shaded cells in (2) when we factor out $N$, we obtain a maximal partial transversal of the Cayley table of $G$ that has length $3 n / 5$.

Groups with an index 2 subgroup miss every second length within the range $\frac{1}{2} n \leqslant \ell<\frac{3}{5} n$. Our next result covers the other half of that range (and more).

Theorem 5.4. Let $L$ be a Latin square of even order $n$ that contains a subsquare $A$ of order $n / 2$. Suppose that $A$ has a near-transversal. Then $L$ contains a maximal partial transversal of length $\ell$ for all $\ell$ such that $\frac{n}{2}<\ell \leqslant \frac{3}{4} n$ and $\ell-\frac{n}{2}$ is even.

Proof. By assumption, $L$ takes the form

$$
\left(\begin{array}{l|l}
A & B \\
\hline C & D
\end{array}\right)
$$

where $A$ and $D$ are subsquares of order $n / 2$ on some symbol set $S$. Also, we are assuming that $A$ has a near-transversal $T$. Let $s$ be the symbol in $S$ that does not appear in $T$. We proceed by constructing a maximal partial transversal of length $\frac{n}{2}+2 x$ for all $1 \leqslant x \leqslant \frac{n}{8}$. Any partial transversal of $D$ of length less than $\frac{n}{4}$ can be extended greedily, so there is a partial transversal $T^{\prime}$ of $D$ of length $x$ that contains the symbol $s$. Let $T^{\prime \prime}$ be formed from $T^{\prime}$ by adding the triples of $T$ whose symbols are not used in $T^{\prime}$. Necessarily $T^{\prime \prime}$ has length $\frac{n}{2}$ and contains the symbols in $S$. We proceed by adding triples from $B$ and $C$ to $T^{\prime \prime}$ whose rows, columns and symbols are distinct from the triples in $T^{\prime \prime}$ until there are exactly $\frac{n}{2}+2 x$ triples. Let $T_{n / 2}=T^{\prime \prime}$ and suppose we have chosen $T_{i} \supseteq T^{\prime \prime}$ such that $T_{i}$ is a partial transversal of length $i$ for some $i<\frac{n}{2}+2 x$. Suppose that no triple of $T_{i}$ is in a particular row $r$ of $B$ (the case when no triple of $T_{i}$ is in a column of $C$ is similar). As $A \cap T_{i}$ contains exactly $\frac{n}{2}-x$ triples, there can be at most $x-1$ triples from $B$ and $x$ triples from $C$ that are in $T_{i}$. Therefore there are at most $2 x-1$ triples from $T_{i}$ that share a symbol with a triple in row $r$ of $B$. Also, as $D \cap T_{i}$ contains exactly $x$ triples, there are at most $2 x-1$ triples from $T_{i}$ that share a column with a triple in row $r$ of $B$. Thus, there are at least $\frac{n}{2}-4 x+2>0$ triples in row $r$ of $B$ that do not share a row, column or symbol with any triple in $T_{i}$, any of which can be added to $T_{i}$ to form a longer partial transversal $T_{i+1}$. Continuing in this way, we can find a partial transversal $T_{\ell} \supseteq T^{\prime \prime}$ of length $\ell=\frac{n}{2}+2 x$. The partial transversal $T_{\ell}$ is maximal, as the only triples not in the same row or column as a triple in $T_{\ell}$ lie in $D$, yet all the symbols of the triples in $D$ are in $T^{\prime \prime}$ and hence also in $T_{\ell}$.

Note that the hypothesis that $A$ has a near transversal is a weak condition in the sense that is conjectured to hold for all Latin squares (see [17]). Together with Theorem 5.2 and Lemma [5.1, Theorem 5.4 shows that the Cayley tables of finite groups with an index 2 subgroup have maximal partial transversals for each length $\ell$ satisfying $\frac{1}{2} n<\ell \leqslant \frac{3}{4} n$ and $\ell \equiv \frac{1}{2} n(\bmod 2)$.

We next find all maximal partial transversal lengths in Cayley tables of small groups. 
Theorem 5.5. The Cayley tables of groups of order $n \leqslant 24$ possess maximal partial transversals of all lengths $\ell$ not forbidden by Theorem 2.2, Theorem 5.1, Corollary 5.1 or Theorem 5.3, except for the following cases:

- Cayley tables of non-cyclic groups of order 8 do not achieve $\ell=5$.

- The Cayley table of $\mathbb{Z}_{9}$ does not achieve $\ell=6$.

- Cayley tables of groups of order $n$ do not achieve $\ell$ when $(n, \ell)$ is one of

$$
(10,6),(11,8),(13,8),(15,10),(17,12),(19,12),(21,13),(21,14),(22,14),(23,14),(23,16) .
$$

- Cayley tables of $\mathbb{Z}_{2} \times \mathbb{Z}_{10}$ and the holomorph $\mathbb{Z}_{5} \rtimes \mathbb{Z}_{4}$ do not achieve $\ell=13$.

- Cayley tables of groups of order 24 other than $\mathbb{Z}_{24}$ and $\mathbb{Z}_{3} \rtimes \mathbb{Z}_{8}$ do not achieve $\ell=15$.

Proof. The result was obtained by two independent computations. By using left and right translation we could assume when convenient that any partial transversal contains the triple $(\varepsilon, \varepsilon, \varepsilon)$, where $\varepsilon$ is the group identity. With this assumption, a simple backtracking search sufficed for all $n \leqslant 16$. Running a partial backtracking search for each group in the range $17 \leqslant n \leqslant 24$ quickly found all lengths $\ell>\frac{2}{3}(n+1)$ that are not forbidden by Theorem 2.2 or Theorem 5.1. Also Corollary 5.1, Theorem 5.3 and Theorem 5.4 resolve the case when $\ell<\frac{3}{5} n$.

For $\frac{3}{5} n \leqslant \ell \leqslant \frac{2}{3}(n+1)$ and $17 \leqslant n \leqslant 24$ we adopted a different strategy for finding a maximal partial transversal $T$ of length $\ell$. We focused first on the "complementary" submatrix $S$ formed by rows and columns of the Cayley table that are not used in $T$. We did not assume that $(\varepsilon, \varepsilon, \varepsilon) \in T$, but rather that $S$ uses row $\varepsilon$ and column $\varepsilon$. We knew that $S$ contains only symbols that are in $T$. So we searched for $(n-\ell) \times(n-\ell)$ submatrices containing at most $\ell$ symbols. We did this by considering all choices for $n-\ell$ rows that included row $\varepsilon$. For each choice of rows, we then built up the set of columns by adding one column at a time, starting with column $\varepsilon$. Most choices quickly violated the bound on the number of symbols in $S$, so this was a fairly quick search. In some cases, such as when $G=\mathbb{Z}_{23}$ and $\ell=14$, there were no viable choices for $S$ and hence we knew that this value of $\ell$ is not achieved for that group. In other cases, there were many viable choices for $S$. For example, when $G=\mathbb{Z}_{23}$ and $\ell=16$ there are $7 \times 7$ submatrices with $13,14,15$ or 16 symbols.

One quick test for viability of a candidate for the submatrix $S$ is as follows. If $S$ contains $m$ symbols where $n-\ell>\frac{2}{3} m$, then Theorem 4.5 implies that $S$ is inside a subsquare of order $m$. If $G$ has no subgroup of order $m$ then this immediately contradicts Lemma 5.1. If $G$ has a subgroup of order $m$ and $m=n / 2$ then the argument at the end of the proof of Theorem 5.3 shows that $\ell-m$ is even (given that $\ell<\frac{3}{4} n$ ). The case when $\ell-m$ is even is handled by Theorem 5.4. In practice, these considerations meant that we never had to do further computations when $n-\ell>\frac{2}{3} m$ and $m>n / 3$.

When a candidate for $S$ was identified and could not be handled by the above arguments, we needed to look for $T$. However, we now knew much more about what we were looking for. We knew exactly which rows and columns $T$ must use (namely the ones that were not in $S$ ). We also knew that $T$ must use all of the symbols that occur in $S$. Typically this either determined the set of symbols in $T$ completely, or almost completely. With this extra information it was usually feasible to determine whether or not $T$ existed by a backtracking search. The hardest case was the example that we have already mentioned, namely $G=\mathbb{Z}_{23}$ and $\ell=16$. For that case, we used one additional piece of information that is available in any abelian group $G$. The sum (in $G$ ) of the symbols within any partial transversal of the Cayley table for $G$ that uses a specified set of rows and columns is just the sum of the indices of those rows and columns (cf. the Delta lemma [17]). This, together with the knowledge that $T$ must contain every symbol in $S$ was enough to eliminate all $7 \times 7$ submatrices of the Cayley table of $\mathbb{Z}_{23}$. For each $7 \times 7$ submatrix there was no feasible set of symbols for $T$ to use. 
In all cases where a maximal partial transversal of a particular length $\ell$ did not exist, our two programs agreed on the reason. Both found the same number (possibly zero) of candidates for $S$. For each candidate they agreed on whether there was a viable set of symbols that might be used in $T$. And if there was, they agreed that there was in fact no $T$.

We can now prove Theorem 1.4. We will say that a group is omniversal or near-omniversal if its Cayley table is omniversal or near-omniversal, respectively.

Proof of Theorem 1.4. First suppose that $G$ is an omniversal group of order $n \geqslant 2$. By Theorem 5.1 , $G$ must be non-abelian and by Theorem 2.1, we know that $n \not \equiv 2(\bmod 4)$. Also the non-abelian groups of order 8 are not omniversal by Theorem 5.5. Our observations thus far ensure that $n \geqslant 12$. In that case, Theorem 5.3 shows that the Cayley table of $G$ has no maximal partial transversal of length $\lfloor n / 2\rfloor+1$, which completes the proof that there are no non-trivial omniversal groups.

Next we suppose that $G$ is a near-omniversal group of order $n \geqslant 2$. From (2), Theorem 5.1 and Theorem 2.2 it follows that $\mathbb{Z}_{3}$ and $\mathbb{Z}_{5}$ are near-omniversal. For odd $n>5$, Theorem 5.3 shows that $G$ misses $\ell=(n+1) / 2$. Together with Theorem 5.1, this eliminates the remaining abelian groups of odd order. Non-abelian groups of odd order are eliminated by Theorem 5.3, given that they have order at least 21.

Having completed the odd case, we may assume that $n$ is even. If $n \equiv 2(\bmod 4)$ then $G$ misses $\ell=n$ by Theorem 2.2. It then follows from Theorem 5.5 that $\mathbb{Z}_{2}, \mathbb{Z}_{6}$ and $D_{6}$ are near-omniversal but that no group of order 10 is. Also for $10<n \equiv 2(\bmod 4)$, Theorem 5.3 rules out $\ell=(n+2) / 2$, showing that $G$ is not near-omniversal.

For $n \equiv 4(\bmod 8)$, Corollary 5.1 rules out $\ell=n / 2$. Hence Theorem 5.1 eliminates $n=4$ and Theorem 5.3 eliminates $12 \leqslant n \equiv 4(\bmod 8)$.

It remains to consider the case when $n$ is divisible by 8 . Theorem 5.5 implies that $D_{8}$ is nearomniversal, but the other groups of order 8 are not, given Theorem 5.1 and Corollary 5.1. Groups of order 16 or 24 are missing $\ell=\frac{n}{2}+1$ by Theorem 5.3. Hence the abelian groups of order 16 or 24 are eliminated by Theorem 5.1. The non-abelian groups of order 16 are near-omniversal, by Theorem 5.5. Also $n \neq 24$, because $\mathbb{Z}_{3} \rtimes \mathbb{Z}_{8}$ misses $\ell=12$ by Corollary 5.1 and all other non-abelian groups of order 24 miss $\ell=15$, by Theorem 5.5 .

Finally, all even groups of order $n>30$ are missing $\ell=\frac{n}{2}+1$ and $\ell=\frac{n}{2}+3$ by Theorem 5.3 , so they are not near-omniversal.

\section{Concluding remarks}

In Theorem 1.2 we settled the existence question for omniversal Latin squares. In Theorem 1.3 we showed that when omniversal Latin squares do not exist, there are usually near-omniversal Latin squares. We also showed in Theorem 5.3 that group tables are increasingly far from omniversal. One direction for future research is raised by Theorem 5.5. It looks like groups (particularly those of odd order) may miss some lengths not predicted by Theorem 5.3. We showed that the $3 n / 5$ in that theorem is best possible, but perhaps there are reasons why some greater lengths are missed. Another question for Cayley tables is how much of a subsquare must be present before we know that the whole subsquare is present. A possible answer was proposed in Conjecture 4.1.

Although we proved in Theorem 3.1 that there exist near-omniversal Latin squares of all orders $n \equiv 2$ $(\bmod 4)$, existence remains open for all large orders $n \not \equiv 2(\bmod 4)$. For any given near-omniversal Latin square $L$ there is one length $\mu_{L}$ of maximal partial transversal that is consistent with (1) but is not obtained in $L$. It may be interesting to consider the set of possible values for $\mu_{L}$ as a function of the order $n$ of $L$. For odd orders, we know of no general restrictions on $\mu_{L}$. However, for even orders, we can say more. Theorem 2.1 implies that for $n \equiv 2(\bmod 4)$ we have $\mu_{L} \in\{n / 2, n\}$. For $n \equiv 0(\bmod 4)$, 
Theorem 5.4 shows that it is impossible to have $\mu_{L} \equiv n / 2(\bmod 2)$ and $n / 2<\mu_{L} \leqslant 3 n / 4$. This is because, if $\mu_{L} \neq n / 2$ then there is a maximal partial transversal of length $n / 2$ and that can only be a transversal of a subsquare of order $n / 2$.

We now briefly examine how these restrictions compare with data for Latin squares of small orders. For this purpose, it is useful to classify Latin squares by viewing them as sets of triples and then using the natural action of $\mathcal{S}_{n} \prec \mathcal{S}_{3}$ on such sets. Orbits of this action are called species (also known as main classes). The action of $\mathcal{S}_{n}$ < $\mathcal{S}_{3}$ preserves the lengths of maximal partial transversals, so it suffices to study one representative of each species.

For $n=6$, there are 10 near-omniversal species and both plausible values for $\mu_{L}$ are realised (see Theorem 1.4 and Theorem 3.1). There are also 2 species of Latin squares which are not near-omniversal (since they are missing maximal partial transversals of both lengths $n / 2$ and $n$ ). For $n=7$ all Latin squares are either omniversal (91 species), near-omniversal with $\mu_{L}=4$ (55 species), or isotopic to the Cayley table of $\mathbb{Z}_{7}$ (which only achieves maximal partial transversals of lengths 5 and 7 ).

For $n=8$, the above considerations show that $\mu_{L} \neq 6$ but it turns out that $\mu_{L}$ cannot be 7 either. The overwhelming majority (283513 of the 283657 species) of Latin squares are near-omniversal with $\mu_{L}=4$. There are 3 species (including that of the dihedral group $D_{8}$ ) that are near-omniversal with $\mu_{L}=5$, and 2 species that are near-omniversal with $\mu_{L}=8$. One example of the latter type (with maximal partial transversals of lengths 4, 5, 6, 7 displayed) is:

\begin{tabular}{|c|c|c|c|}
\hline $\begin{array}{llll:llll}0 & 1 & 2 & 3 & 4 & 5 & 6 & 7\end{array}$ & $\begin{array}{llll:llll}0 & 1 & 2 & 3 & 4 & 5 & 6 & 7\end{array}$ & $\begin{array}{llll:llll}0 & 1 & 2 & 3 & 4 & 5 & 6 & 7\end{array}$ & $\begin{array}{llll:llll}0 & 1 & 2 & 3 & 4 & 5 & 6 & 7\end{array}$ \\
\hline $\begin{array}{llll:llll}1 & 0 & 3 & 2 & 5 & 4 & 7 & 6\end{array}$ & $\begin{array}{llll:llll}1 & 0 & 3 & 2 & 5 & 4 & 7 & 6\end{array}$ & $\begin{array}{llll:llll}1 & 0 & 3 & 2 & 5 & 4 & 7 & 6\end{array}$ & $\begin{array}{llllllll}1 & 0 & 3 & 2 & 5 & 4 & 7 & 6\end{array}$ \\
\hline \begin{tabular}{llll|llll}
2 & 3 & 0 & 1 & 6 & 7 & 4 & 5
\end{tabular} & $\begin{array}{llll:lllll}2 & 3 & 0 & 1 & 6 & 7 & 4 & 5\end{array}$ & $\begin{array}{llll:llll}2 & 3 & 0 & 1 & 6 & 7 & 4 & 5\end{array}$ & $\begin{array}{llllllll}2 & 3 & 0 & 1 & 6 & 7 & 4 & 5\end{array}$ \\
\hline $\begin{array}{llllllll}3 & 2 & 1 & 0 & 7 & 6 & 5 & 4\end{array}$ & $\begin{array}{llllllll}3 & 2 & 1 & 0 & 7 & 6 & 5 & 4\end{array}$ & $\begin{array}{llllllll}3 & 2 & 1 & 0 & 7 & 6 & 5 & 4\end{array}$ & $\begin{array}{llllllll}3 & 2 & 1 & 0 & 7 & 6 & 5 & 4\end{array}$ \\
\hline $\begin{array}{llllllll}4 & 5 & 6 & 7 & 3 & 2 & 1 & 0\end{array}$ & $\begin{array}{llllllll}4 & 5 & 6 & 7 & 3 & 2 & 1 & 0\end{array}$ & $\begin{array}{llllllll}4 & 5 & 6 & 7 & 3 & 2 & 1 & 0\end{array}$ & $\begin{array}{llllllll}4 & 5 & 6 & 7 & 3 & 2 & 1 & 0\end{array}$ \\
\hline $\begin{array}{llll:llll}5 & 4 & 7 & 6 & 0 & 1 & 2 & 3\end{array}$ & $\begin{array}{llll:llll}5 & 4 & 7 & 6 & 0 & 1 & 2 & 3\end{array}$ & $\begin{array}{llll:llll}5 & 4 & 7 & 6 & 0 & 1 & 2 & 3\end{array}$ & $\begin{array}{llllllll}5 & 4 & 7 & 6 & 0 & 1 & 2 & 3\end{array}$ \\
\hline $\begin{array}{llll:llll}6 & 7 & 4 & 5 & 2 & 3 & 0 & 1\end{array}$ & $\begin{array}{lllllllll}6 & 7 & 4 & 5 & 2 & 3 & 0 & 1\end{array}$ & \begin{tabular}{llll|llll}
6 & 7 & 4 & 5 & 2 & 3 & 0 & 1
\end{tabular} & $\begin{array}{llllllll}6 & 7 & 4 & 5 & 2 & 3 & 0 & 1\end{array}$ \\
\hline $\begin{array}{llll:llll}7 & 6 & 5 & 4 & 1 & 0 & 3 & 2\end{array}$ & $\begin{array}{llll:llll}7 & 6 & 5 & 4 & 1 & 0 & 3 & 2\end{array}$ & $\begin{array}{llll:llll}7 & 6 & 5 & 4 & 1 & 0 & 3 & 2\end{array}$ & $\begin{array}{llllllll}7 & 6 & 5 & 4 & 1 & 0 & 3 & 2\end{array}$ \\
\hline
\end{tabular}

For order 8, there are 105 species of omniversal Latin squares, and 34 species that are missing at least two lengths of maximal partial transversals. Of those 34 species, only one is missing three lengths. That species contains the following Latin square, which only has maximal partial transversals of lengths 6 and 7 (as shown by the highlighted cells)

\begin{tabular}{|llll:llll|}
\hline 0 & 1 & 2 & 3 & 4 & 5 & 6 & 7 \\
1 & 2 & 3 & 0 & 5 & 6 & 7 & 4 \\
2 & 3 & 0 & 1 & 6 & 7 & 4 & 5 \\
3 & 0 & 1 & 2 & 7 & 4 & 5 & 6 \\
\hdashline & 6 & 5 & 4 & 0 & 3 & 2 & 1 \\
6 & 5 & 4 & 7 & 3 & 2 & 1 & 0 \\
5 & 4 & 7 & 6 & 2 & 1 & 0 & 3 \\
4 & 7 & 6 & 5 & 1 & 0 & 3 & 2 \\
\hline
\end{tabular}

\begin{tabular}{|llll:llll|}
\hline 0 & 1 & 2 & 3 & 4 & 5 & 6 & 7 \\
1 & 2 & 3 & 0 & 5 & 6 & 7 & 4 \\
2 & 3 & 0 & 1 & 6 & 7 & 4 & 5 \\
3 & 0 & 1 & 2 & 7 & 4 & 5 & 6 \\
\hdashline 7 & 6 & 5 & 4 & 0 & 3 & 2 & 1 \\
6 & 5 & 4 & 7 & 3 & 2 & 1 & 0 \\
5 & 4 & 7 & 6 & 2 & 1 & 0 & 3 \\
4 & 7 & 6 & 5 & 1 & 0 & 3 & 2 \\
\hline
\end{tabular}

Examples like this prompt another research direction, which is to look for Latin squares that have maximal partial transversals of as few different lengths as possible. Note that it follows from [2, Thm 13] that almost all large Latin squares obtain only sublinearly many lengths, and hence are very far from being omniversal. In contrast, Theorem 5.4 implies that the presence of a maximal partial transversal of length $n / 2$ in a Latin square of (even) order $n$ necessitates the presence of maximal partial transversals of linearly many lengths. 


\section{References}

[1] G. B. Belyavskaya and A. F. Russu, Partial admissibility of quasigroups (Russian), Quasigroups and combinatorics, Mat. Issled. Vyp. 43 (1976), 50-58, 204.

[2] D. Best, T. Marbach, R. J. Stones and I. M. Wanless, Covers and partial transversals of Latin squares, Des. Codes Cryptogr., 87(5) (2019), 1109-1136.

[3] J. N. Bray, Q. Cai, P. J. Cameron, P. Spiga and H. Zhang, The Hall-Paige conjecture, and synchronization for affine and diagonal groups, J. Algebra 545 (2020), 27-42

[4] J. M. Browning, P. J. Cameron and I. M. Wanless, Bounds on the number of small Latin subsquares, J. Combin. Theory Ser. A 124 (2014), 41-56.

[5] A. Drápal, How far apart can the group multiplication tables be?, European J. Combin. 13 (1992), 335-343.

[6] A. B. Evans, Orthogonal Latin squares based on groups, Develop. Math. 57, Springer, Cham, 2018.

[7] A. B. Evans, Maximal partial transversals in a class of Latin squares, Australasian J. Combin. 73 (2019), 179-199.

[8] L. Goddyn and K. Halasz, All group-based Latin squares possess near transversals, J. Combin. Des. 28 (2020), 358-365.

[9] Y. O. Hamidoune, Extensions of the Scherk-Kemperman theorem, J. Combin. Theory Ser. A 117 (2010), 974-980.

[10] G. Ivanyos, F. Le Gall, Y. Yoshida, On the distance between non-isomorphic groups, European J. Combin. 33 (2012), 474-476.

[11] J. H. B. Kemperman, On complexes in a semigroup. Nederl. Akad. Wetensch. Proc. Ser. A. 59 (1956), 247-254.

[12] M. Kneser, Abschätzung der asymptotischen Dichte von Summenmengen. (German) Math. Z. 58 (1953), 459-484.

[13] J. E. Olson, On the sum of two sets in a group, J. Number Theory 18 (1984), 110-120.

[14] L. J. Paige, A note on finite abelian groups, Bull. Amer. Math. Soc. 53 (1947), 590-593.

[15] H. J. Ryser, A combinatorial theorem with an application to Latin rectangles, Proc. Amer. Math. Soc. 2, (1951) 550-552.

[16] P. Vojtěchovský and I. M. Wanless, Closest multiplication tables of groups, J. Algebra 353 (2012), 261-285.

[17] I. M. Wanless, Transversals in Latin squares: a survey, in "Surveys in Combinatorics 2011", 403437, London Math. Soc. Lecture Note Ser. 392, Cambridge Univ. Press, 2011. 